\title{
A web-based survey on academic fields of interest among the Biomedical Informatics trainees and Health Informaticians in Sri Lanka
}

\author{
S. Irfaan $^{1 *}$, C.M. Manawadu ${ }^{1}$, S.T. Pathirana ${ }^{1}$, F.S. Mubarak ${ }^{1}$, M.I.N. Ikram¹, \\ P. Siribaddana ${ }^{2}$ \\ 1 Postgraduate Institute of Medicine, University of Colombo, Sri Lanka \\ *bmi9group3@gmail.com \\ https://orcid.org/0000-0002-9725-0377 \\ ABSTRACT
}

\begin{abstract}
Introduction: Biomedical Informatics is a rapidly growing discipline, which intersects the fields of Information Communication Technology, Medicine and Biology. Given the multidisciplinary and rapidly evolving nature of this field of study, trainees and graduates may possess a variety of academic and research interests, complicating the task of training and curriculum development. The aim of this study is to identify the key interest areas among the trainees of existing post graduate programmes in Biomedical and Health Informatics, conducted by the Post Graduate Institute of Medicine (PGIM), Sri Lanka in view of identifying their key learning needs.
\end{abstract}

Methods: A web-based survey was conducted among current MSc trainees of Biomedical Informatics and MD trainees of Health Informatics attached to Post Graduate Institute of Medicine, University of Colombo, Sri Lanka. Participants were asked to rate academic interest for given fields and to rate interest for the tools/areas in which they want to build their competency. The rating was done using a Likert scale. Gathered data was analysed using descriptive statistics.

Results: There were 40 responders (Response rate $-47.1 \%$ ). Forty five percent had MBBS as their highest educational qualification and the rest had MSc, MD or BDS. Out of the 17 Academic areas checked for, Clinical Informatics (median $=4.4)$, followed by the Decision Support Systems and Artificial Intelligence $($ median $=4.2$ ) are the areas which had drawn the highest level of academic interest. The lowest level of academic interest was identified in Bioinformatics (median $=3.1$ ). Electronic Health Record systems (median $=4.375$ ) is the area in which most of the participants want to build their competency and least preference was given to BLAST (Basic Local Alignment Search Tool) (median $=2.75$ ) out of the 22 tools/areas checked for.

Conclusions: The interests and needs of the trainees of these courses are diverse, which warrants constant curriculum reviews. The areas highlighted in this study could be given priority when revising curriculums and allocating resources for the MSc and MD courses.

Keywords: Academic interest, Biomedical Informatics, Health Informatics, Health Informaticians 